\title{
Encoding Genetic Information and Catalyzing Chemical Reactions: The Multifunctional Roles of Nucleic Acids
}

Li A*

Genetics and Aging Research Unit, Department of Neurology, Massachusetts General Hospital and Harvard Medical School, Charlestown, MA 02129, USA

*Corresponding author: Airong Li, Genetics and Aging Research Unit, Department of Neurology, Massachusetts General Hospital and Harvard Medical School, Charlestown, MA 02129, USA, Tel: 1-617-724-9397; Fax: 36-1-225-3899; E-mail: ali3@mgh.harvard.edu

Rec date: January 25, 2015, Acc date: January 27, 2015, Pub date: January 30, 2015

Copyright: (c) $2015 \mathrm{Li} \mathrm{A}$. This is an open-access article distributed under the terms of the Creative Commons Attribution License, which permits unrestricted use, distribution, and reproduction in any medium, provided the original author and source are credited.

\section{Editorial}

Nucleic acids are polymolecules that include DNA (Deoxyribonucleic acid) and RNA (ribonucleic acid), which are found in abundance in all living things and essential for all known forms of life. Nucleic acids were first discovered in 1869 by Friedrich Miescher, who later also proposed that nucleic acids could be involved in heredity.

DNA exist as a pair of molecules that are held tightly together, called the double helix structure, which was discovered in 1950s by James Watson and Francis Crick [1], who won the Nobel Prize in Physiology or Medicine 1962 for their discoveries concerning the molecular structure of nucleic acids. Each strand of DNA in the double helix can serve as a pattern for duplicating the sequence of bases, which is critical when cells divide and allows each new cell to have an exact copy of the DNA present in the old cell, or the genetic information transfer in living material. Most DNA is located in the cell nucleus but a small amount of DNA can also be found in the mitochondria.

Living organisms are dependent on nucleic acids' function in encoding, transmitting and expressing genetic information for storing and transmitting hereditary via protein synthesis. RNA controls and regulates many aspects of protein synthesis according to the instructions encoded within a cell's DNA through the processes of transcription and translation. During the process of transcription, the information stored in a gene's DNA is transferred to messenger RNA (mRNA) in the cell nucleus, which carries the information, or message, from the DNA out of the nucleus into the cytoplasm. During the process of translation, the mRNA interacts with ribosome in the cytoplasm to decode the sequence of mRNA bases for amino acid synthesis and protein build. Then, the transfer RNA (tRNA) assembles the protein, one amino acid at a time until the ribosome encounters a "stop" codon. Proteins are responsible for catalyzing the biochemical reactions that are required to provide food and energy and for all forms of movement for every organism.

Chemical reactions in living organisms taking place in a living cell are dependent on biological catalysts or enzymes to facilitate. These biocatalysts or enzymes increase reaction rates by some million-fold but without being consumed or changed themselves. For example in the liver there are two enzymes-alcohol dehydrogenase $(\mathrm{ADH})$ and aldehyde dehydrogenase (ALDH) that help break apart the alcohol molecule, making it possible to eliminate it from the body.

While the critical roles of nucleic acids in encoding genetic information have been known for over 60 years, the function of nucleic acids as biological catalysts has only been recognized since 1980s. Sidney Altman and Thomas Cech independently demonstrated that during the process of the genetic code of the DNA transcribed into
RNA, a shearing and splicing of the RNA molecules is required apart from the actual transcription, and discovered RNA enzymes or ribozymes in the early 1980s [2]. Subsequent development has been rapid and today close to a hundred ribozymes are known. These ribozymes were found in the intron of an RNA transcript, which removed itself from the transcript, as well as in the RNA component of the RNase P complex, which is involved in the maturation of pretRNAs [2]. Altman and Cech were awarded the Nobel Prize in chemistry in 1989 for their discovery of catalytic properties of RNA. The discovery of catalytic RNA indicates that it is likely that the RNA molecules were the first biomolecules to contain both the genetic information and play a role as biocatalysts. This concept has influenced profoundly on our understanding of how life on earth began and developed.

DNA-based hybrid catalysis was introduced only ten years ago [3]. The catalytic properties of DNA hybrid catalysts are governed by the disposition of the metal-binding site in the DNA duplex, the size of catalytic cavity, and the composition of nucleobases in the catalytic pocket. This concept has been applied successfully in a number of archetypal $\mathrm{C}-\mathrm{C}$ and $\mathrm{C}-\mathrm{O}$ bond forming reactions, such as the copper (II) catalyzed Diels-Alder, (oxa)- Michael addition and Friedel-Crafts alkylation reactions [3]. During the last five years, DNA-based asymmetric catalysis is rapidly emerging as a promising new concept in catalysis and as a novel tool to be applied successfully in a variety of catalytic enantioselective reactions. A DNA-based catalyst comprises a transition metal complex based on a non-chiral ligand that is brought into close proximity of the DNA helix. As a result the catalyzed reaction takes place in, or very close to, the DNA helix, which allows the chirality of DNA to be transferred onto the reaction, resulting in products that have an excess in one of their enantiomers [4]. Thus DNA has emerged as an attractive scaffold for the design of novel enantioselective catalysts.

The discovery of the functional roles of RNA and DNA altered the central dogma of the biosciences, and provides evidence that nucleic acids are the molecules of not only heredity but also biocatalysts, or enzymes. These new concepts have also provided a new tool for gene technology.

XNAs, or xeno nucleic acids, are synthetic genetic polymers that have shown potential for either chemical and/or enzymatic replication, which was first proposed by Herdewijn and Marliere in 2009 [5]. Advances in nucleotide chemistry and polymerase engineering enable the synthesis and replication of XNAs. Catalysis by nucleic acids (and by biopolymers in general) requires as a minimum the presence of chemically functional groups and a framework for their precise arrangement. The backbones XNAs based on congeners of the canonical ribofuranose share with RNA and DNA a capacity for 
Citation: Li A (2015) Encoding Genetic Information and Catalyzing Chemical Reactions: The Multifunctional Roles of Nucleic Acids. Hereditary Genet 4: e113. doi:10.4172/2161-1041.1000e113

Page 2 of 2

heredity, evolution and the ability to fold into defined threedimensional structures and forming ligands [6]. Notably, very recently scientists have isolated synthetic XNAs that can also carry out chemical reactions [7]. Holliger's team generated large libraries of random XNA sequences and screened the libraries for molecules that could carry out reactions, and they found that XNAs can act as enzymes to catalyze these reactions. This means that life could be based on other molecules besides DNA and RNA, and XNAs also provide a new potential source of enzymes that can avoid natural degradation pathways [7]. Collectively, the exploration of XNA sequence space promises to deliver new ligands, catalysts and materials, which may facilitate the discovery of novel therapeutics that could carry out chemical reactions in the human body while avoiding nuclease enzymes that target RNA and DNA.

\section{References}

1. Watson JD, Crick FH (1953) Molecular structure of nucleic acids; a structure for deoxyribose nucleic acid. Nature 171: 737-738.
2. Kruger K, Grabowski PJ, Zaug AJ, Sands J, Gottschling DE, et al. (1982) Self-splicing RNA: autoexcision and autocyclization of the ribosomal RNA intervening sequence of Tetrahymena. Cell 31: 147-157.

3. Rioz-Martínez A, Roelfes G (2015) DNA-based hybrid catalysis. Curr Opin Chem Biol 25C: 80-87.

4. Boersma AJ, Megens RP, Feringa BL, Roelfes G (2010) DNA-based asymmetric catalysis. Chem Soc Rev 39: 2083-2092.

5. Herdewijn P, Marlière P (2009) Towards safe genetically modified organisms through the chemical diversification of nucleic acids. Chem Biodivers 6: 791-808.

6. Pinheiro VB, Holliger P (2012) The XNA world: progress towards replication and evolution of synthetic genetic polymers. Curr Opin Chem Biol 16: 245-252.

7. Taylor AI, Pinheiro VB, Smola MJ, Morgunov AS, Peak-Chew S, et al. (2014) Catalysts from synthetic genetic polymers. Nature. 